\title{
Non-parallel transcriptional divergence during parallel adaptation
}

\author{
Eva Fischer ${ }^{1}$, Youngseok Song ${ }^{2}$, Kimberly Hughes ${ }^{3}$, Wen Zhou ${ }^{2}$, and Kim Hoke ${ }^{2}$ \\ ${ }^{1}$ University of Illinois at Urbana-Champaign \\ ${ }^{2}$ Colorado State University \\ ${ }^{3}$ Florida State University
}

July 20, 2020

\begin{abstract}
How underlying mechanisms bias evolution toward predictable outcomes remains an area of active debate. In this study, we leveraged phenotypic plasticity and parallel adaptation across independent lineages of Trinidadian guppies (Poecilia reticulata) to assess the predictability of gene expression evolution during parallel adaptation. We observed substantial gene expression plasticity as well as the evolution of expression plasticity itself across populations. Genes exhibiting expression plasticity within populations were more likely to also differ in expression between populations, with the direction of expression divergence more likely to be opposite than in the same direction as plasticity. While we also found more overlap than expected by chance in genes differentially expressed between high- and low-predation populations from different lineages, the majority of differentially expressed genes were not shared between distinct evolutionary lineages. Our data suggest alternative transcriptional configurations associated with shared phenotypes, highlighting a role for transcriptional flexibility associated with parallel phenotypic evolution in a species known for rapid adaptation.
\end{abstract}

\section{Introduction}

Phenotypic evolution is biased by the mechanisms that link genetic variation to phenotypic variation, i.e. the genotype-phenotype map (Alberch, 1991; Pigliucci, 2010). Mechanisms linking genotype to phenotype are characterized by both robustness and flexibility. Robust developmental processes buffer phenotypes from noise such that multiple transcriptional, biochemical, or cellular network configurations give rise to similar organismal phenotypes, while flexibility in these processes allows for phenotypic plasticity in response to environmental conditions (Marder \& Goaillard, 2006; Siegal \& Leu, 2014; Wagner, 2011). Though contrasting, both phenomena are ultimately bounded by phenotypic, developmental, genetic, and mechanistic constraints. The complex nature of the genotype-phenotype map and the existence of 'many-to-one' mappings across hierarchical levels of biological organization make it challenging to understand how mechanistic properties facilitate or constrain adaptive evolution. Here, we combine studies of phenotypic plasticity and parallel adaptation to assess how mechanistic biases shape evolutionary trajectories across timescales.

Studies of parallel adaptation can reveal biases in mechanisms of evolution by asking whether similar mechanism are associated with repeated, independent evolutionary transitions. Similar mechanisms underlying parallel evolutionary transitions suggest the genotype-phenotype map is constrained by limits on the possible ways to construct adaptive phenotypes (Losos, 2011). In contrast, non-shared mechanisms suggest that mechanistic flexibility may facilitate evolution by providing multiple alternative 'solutions' to a given adaptive problem and providing alternative paths by which organisms can reach adaptive peaks (Badyaev \& Morrison, 2018; Drion, O'Leary, \& Marder, 2015; Grashow, Brookings, \& Marder, 2009). Such mechanistic flexibility may allow organisms to more easily reach the same adaptive peak from alternative starting points, and additionally buffer organisms from pleiotropic effects via compensatory mechanisms that rely on the many-to-one mappings created by these alternative paths. 
One potential consequence of the biological robustness generated by many-to-one mappings is that variation in the function of only a small number of key genes will alter organismal phenotypes while variation in most genes has no phenotypic consequences (Yang, Maclean, Park, Zhao, \& Zhang, 2017), either because variation does not propagate to higher levels of organization and/or because variation is compensatory. In this case, phenotypic evolution may rely on a limited number of mechanistic paths, repeatedly targeting those mechanisms that that yield the greatest phenotypic responses with the smallest pleiotropic costs. Indeed, compelling examples demonstrate that similar phenotypes share underlying neural, physiological, molecular, and/or genetic mechanisms, even across highly divergent taxa (e.g. Insel and Young, 2000; Manceau et al., 2010; Pankey et al., 2014; Rosenblum et al., 2010). In contrast, other studies demonstrate flexibility in underlying mechanisms suggesting that different mechanistic 'solutions' can give rise to shared phenotypes in closely related species, among populations of the same species, or even among individuals of the same population (e.g. Abouheif and Wray, 2002; Crawford and Oleksiak, 2007; Drion et al., 2015; Grashow et al., 2009; Mandic et al., 2018). Nevertheless, even if mechanistic flexibility is a common feature of robust biological networks, shared genetic background or patterns of pleiotropy in a lineage may still direct evolutionary paths toward predictable mechanistic pathways, especially at long evolutionary timescales (Gompel \& Prud'homme, 2009; Stern \& Orgogozo, 2008). Empirical evidence for both shared and distinct mechanisms underlying parallel phenotypic evolution leaves open the question of when and why either pattern dominates.

One feature of biological networks that may channel divergence into particular paths is environmentally induced plasticity. First, both plastic and evolutionary processes may rely on those mechanistic paths that yield the greatest phenotypic responses. In this case, we expect plastic and evolutionary responses to share underlying mechanisms, although they may not act in the same direction. Theory predicts that plastic and evolutionary changes will be in the same direction when plasticity in a novel environment is adaptative, increasing immediate survival and allowing time for evolutionary divergence via co-option of mechanisms involved in environmentally induced responses (Baldwin, 1896; Ghalambor, McKay, Carroll, \& Reznick, 2007; Lande, 2009; West-Eberhard, 2003). In contrast, plastic and evolutionary changes will be in opposite directions when plasticity in a novel environment is non-adaptive thereby increasing the strength of selection or when plastic responses 'overshoot' adaptive optima and are compensated by selection (Conover, Duffy, \& Hice, 2009; Ghalambor et al., 2007; Grether, 2005; Velotta \& Cheviron, 2018). Importantly, plasticity may facilitate adaptation under either scenario and empirical studies document both patterns. In addition, plastic and evolutionary processes may rely on shared mechanistic paths because the mechanisms mediating phenotypic plasticity promote the accumulation of cryptic genetic variation that is released under new environmental conditions, thereby fostering associations between plasticity and divergence (Draghi \& Whitlock, 2012; Espinosa-Soto, Martin, \& Wagner, 2011). Finally, while associations between phenotypic plasticity and evolution have primarily been considered in the context of phenotypic change, associations between developmental plasticity and genetic divergence at the level of underlying mechanisms could also arise if initially plastic compensatory or homeostatic responses to maintain, rather than alter, organism level phenotypes become genetically fixed as adaptation proceeds (Velotta \& Cheviron, 2018), in which case we may also expect the evolution of plasticity itself.

In the present study, we take advantage of parallel phenotypic evolution in independent lineages of Trinidadian guppies (Poecilia reticulata) to explore patterns of flexibility and constraint in transcriptional mechanisms mediating repeated adaptation. We compare whole-brain gene expression patterns based on developmental experience and genetic background to test four hypotheses: (1) that genes with significant expression plasticity are more likely to show genetic divergence in expression, (2) that the direction of plastic responses predicts the direction of genetic divergence, (3) that gene expression plasticity itself evolves, and (4) that parallel phenotypic adaptation across independent lineages relies on shared gene expression changes.

Guppies as a model system

Guppies have become a model system in ecology and evolutionary biology due to repeated, independent adaptation of natural populations to distinct predation environments (Endler, 1995; Haskins, Haskins, McLaugh- 
lin, \& Hewitt, 1961; D. Reznick, Butler IV, \& Rodd, 2001). In Trinidad, high- and low-predation population pairs from different river drainages represent independent evolutionary lineages (Barson, Cable, \& Van Oosterhout, 2009; Fraser, Künstner, Reznick, Dreyer, \& Weigel, 2015; Gilliam, Fraser, \& Alkins-Koo, 1993; Willing et al., 2010) in which colonization of low-predation environments has led to parallel, adaptive changes in life history traits, morphology, and behavior (Endler, 1995; Magurran, 2005; D. A. Reznick, Bryga, \& Endler, 1990; D. Reznick et al., 2001; D. N. Reznick, 1997). For example, low-predation guppies shoal less tightly (Huizinga, Ghalambor, \& Reznick, 2009; Magurran \& Seghers, 1990b, 1991), escape more slowly (Ghalambor, Reznick, \& Walker, 2004), are slower to re-commence movement following a predator encounter (Elvidge, Ramnarine, \& Brown, 2014; Harris, Ramnarine, Smith, \& Pettersson, 2010), and perform fewer predator inspections (Magurran \& Seghers, 1990a, 1994) than their high-predation counterparts.

Building on decades of work comparing high- and low-predation populations from the wild, more recent laboratory breeding designs have disentangled genetic and environmental influences of predation on phenotypic differences. These studies demonstrate that a combination of genetic and environmental influences shape guppy life history (Torres Dowdall et al., 2012), morphology (Fischer, Soares, Archer, Ghalambor, \& Hoke, 2013; C. A. Handelsman, Ruell, Torres-Dowdall, \& Ghalambor, 2014; Ruell et al., 2013; TorresDowdal, Handelsman, Reznick, \& Ghalambor, 2012), physiology (Fischer, Harris, Hofmann, \& Hoke, 2014; Corey A. Handelsman et al., 2013), and behavior (Fischer, Ghalambor, \& Hoke, 2016b; Huizinga et al., 2009; Torres-Dowdal et al., 2012). Yet despite many years of work at the level of organism level phenotypes, the mechanisms underlying adaptive phenotypic differences in guppies remain largely unexplored. A single study characterized brain gene expression in multiple populations from the same lineage during the earliest stages of adaptation ( $\sim 3$ generations after colonization of low-predation environments) and found a negative relationship between phenotypic plasticity and adaptive divergence (Ghalambor et al., 2015).

In the current study, we compare the effects of genetic background (high- versus low-predation populations) and developmental environment (rearing with and without predator cues) on brain gene expression patterns in two parallel, independent evolutionary lineages of guppies. These lineages diverged at least 600,000 years ago, with subsequent, more recent colonization of low-predation environments by high-predation ancestors within each river drainage (Fajen \& Breden, 1992; Willing et al., 2010). We here compare whole-brain gene expression patternswithin and between lineages based on evolutionary history with and rearing with predators. We focus on gene expression specifically in the brain because the previous gene expression study in guppies used brain tissue (Ghalambor et al., 2015) and because of previous evidence for behavioral plasticity in our focal populations (Fischer et al., 2016b).

To test our hypotheses we (1) quantify the extent of overlap in genes exhibiting significant expression plasticity as well as genetic expression divergence within each drainage, (2) examine associations in the direction of plastic and genetic expression differences, (3) ask whether expression plasticity itself evolves, and (4) assess whether parallel phenotypic adaptation across lineages relies on shared gene expression mechanisms. First, if developmental plasticity does indeed predict genetic divergence, we expect a within-lineage association in gene identity and/or expression direction among those genes showing plastic and genetic expression differences. Second, if gene expression plasticity itself evolves, we expect the extent and/or direction of plastic responses to differ between genetic backgrounds. Finally, if only a few gene expression configurations (i.e. mechanistic paths) can give rise to shared adaptive behavioral phenotypes, we expect parallel adaptation to low-predation habitats to be characterized by parallel evolution in a set of genes that are largely shared between lineages. In contrast, if transcriptional mechanisms are flexible, we expect gene expression divergence in largely non-overlapping gene sets. Taken together, our results allow us to assess the flexibility of transcriptional mechanisms of adaptation across timescales - from developmental plasticity, to genetic divergence, to parallel adaptation across lineages.

\section{Material and Methods}

Fish collection and rearing

We established lab populations of guppies from high- and low-predation populations pairs collected from 
the Aripo and Quare river drainages in 2012 and 2014, respectively. To maintain the genetic variation of the original wild-caught fish, we established 20 - 30 unique family lines for each population (i.e., for each generation a single female from each family was crossed to a single male from another family) (D. N. Reznick \& Bryga, 1987). We used second-generation lab born fish from these unique family lines in this study to minimize environmental and maternal effects. At birth, we split second-generation siblings into rearing environments with (pred+) or without (pred-) predator chemical cues, and they remained in these environments until the completion of the experiment (as in Fischer et al., 2016). We used only mature males in this study. To maximize the range of genetic variation captured among focal fish, all males in a given experimental group (i.e. population and rearing environment) were from distinct families; however, our splitbrood design allowed us to simultaneously leverage genetic similarity among siblings and control for the effects of genetic background on developmental responses. Figure 1 provides an overview of our experimental design and interpretation of comparisons.

All guppies were individually housed in 1.5 liter tanks on a 12:12 hour light cycle at Colorado State University. Fish were fed a measured food diet once daily, receiving Tetramin tropical fish flake paste and hatched Artemia cysts on an alternating basis. Prior to tissue collection for this study, behavioral and hormone data were collected in an identical manner for all fish and these results are reported elsewhere (Fischer et al., 2016b). All experimental methods were approved by the Colorado State University Animal Care and Use Committee (Approval \#12-3818A).

\section{Tissue collection and processing}

We collected brain tissue from the Aripo and Quare lineage males described above in 2013 and 2015, respectively. To standardize any effects of recent experience, behavior, and circadian rhythm on gene expression, we collected whole brains within 10 minutes of lights-on. We interpret our transcriptional data as baseline, in the sense that fish were minimally stimulated prior to tissue collection, and expression levels should therefore reflect genetic background and developmental experience more strongly than responses to immediate environmental context. Fish were anesthetized by immersion in ice water followed by rapid decapitation. Whole brains were removed, flash frozen in liquid nitrogen, and stored at -80degC until further processing. Tissue collection took $<2$ minutes, rapid enough to minimize changes in gene expression responses to handling and dissection.

We extracted total RNA from brain tissue using the Qiagen RNeasy Lipid Tissue Mini Kit (Qiagen, Germany) following manufacturer guidelines. We prepared separate sequencing libraries for each individual using the NEBNext Ultra RNA Library Prep Kit for Illumina (New England Biolabs, Massachusetts, USA) following manufacturer instructions. Libraries were sequenced on an Illumina HiSeq 2000 at the Florida State University College of Medicine Translational Science Laboratory (Tallahassee, Florida) in May 2014 (Aripo dataset) and January 2016 (Quare dataset). For the Aripo dataset, 40 samples (N=10 per group) were combined with unique barcodes into eight samples per pool and each pool was sequenced on a single lane. For the Quare dataset, 60 samples ( $N=12-14$ per group) were combined into three pools with 20 samples per pool and each pool was sequenced in two separate lanes. Blocks of individuals run in the same week were processed and sequenced together, and experimental groups were balanced across sequencing lanes.

\section{Transcript abundance estimation}

We received 1.45 billion 100-bp paired-end reads that passed the HiSeq quality filter, averaging 14.5 million reads per sample. We used Rcorrector to amend Illumina sequencing errors (Song \& Florea, 2015), and removed adapter sequences and trimmed reads for high-quality sequence using Trim Galore! (Babraham Bioinformatics, Babraham Institute). Following developer recommendations, we used a quality score of 33, a stringency of 5 , and a minimum read length of 36bp. We aligned corrected, trimmed reads from both datasets to the guppy genome (http://uswest.ensembl.org/Poecilia_reticulata; downloaded November 2019) and estimated transcript abundance using STAR (Dobin et al., 2013) with default parameters. On average, 91\% (range: $88.8 \%-94.2 \%$ ) of sequences per individual mapped to the guppy genome assembly.

Data filtering and screening 
Preliminary cluster analyses revealed retinal contamination in a subset of our Aripo dataset brain samples. While opsins are expressed at low levels in the brain, the very high expression levels $(>10,000$ copies) in three samples pointed to retinal contamination. To deal with this issue, we devised a sample filtering and screening procedure to remove genes in which expression differences between samples were likely dominated by retinal contamination. Briefly, we first filtered genes with low expression, then we used contigs annotated as known retinal genes (Rhodopsin, red/green-sensitive opsins, blue-sensitive opsins) as seed contigs to identify other contamination-related transcripts based on high positive correlations of expression levels with seed genes. We calculated the gene-wise sum of correlations between candidate genes and seed genes and performed multiple hypothesis testing using a false discovery rate (FDR) controlling procedure. The nominal level of FDR was set to $\alpha=0.2$ to remove presumptive contaminant contigs. Using this approach, we identified 1,559 contigs as presumptive retina-enriched genes ( $\sim 3 \%$ of all contigs in our final assembly) which we removed from both datasets in all subsequent analyses (Table S1). More detailed descriptions of statistical procedures are in the Supplemental Methods.

\section{Differential expression analysis}

Due to differences in timing of fish rearing, sample processing, and sequencing, we did not combine Aripo and Quare datasets for statistical analysis. We instead performed analysis in an identical fashion for both drainages and subsequently conducted separate analyses to test hypotheses concerning mechanistic parallelism across drainages (see below). Standard differential expression analysis packages could not accommodate the random effects in our experimental design (i.e. family and week; see below) but including these effects improved model fit and reflected our split-brood experimental design. Upon fitting a generalized linear mixed model with negative binomial link to each data set (detailed in the Supplemental Methods), a portion of genes had statistically significant random effects due to either family or week or both. Model comparisons between the generalized linear model without random effects and the generalized linear mixed model including week and family effects supported incorporating random effects, as likelihood ratio tests indicated better fit of the mixed models for 391 out of 19,004 genes in the Aripo dataset and 1,194 out of 19,902 genes in the Quare dataset. For consistency, we performed differential expression analysis using generalized linear mixed models with random effects for all genes as further detailed in the Supplemental Materials.

We normalized read counts using DESeq2 in R (Love, Huber, \& Anders, 2014) and performed differential expression analysis using the lme4 package in R (github.com/lme4). Normalized count data were modeled using a generalized linear mixed model with negative binomial distribution. We included population of origin, rearing environment, and their interaction as fixed effects. In addition, we included family (siblings split across rearing environments) and week (tissue was collected from animals in balanced blocks across multiple weeks) as random effects. A Wald's test provided p-values for main effects and interaction effects for each gene (Lehmann \& Romano, 2005). We adjusted p-values for multiple hypothesis testing using a direct approach for FDR correction (Storey, 2002) as implemented in the fdrtool package in R (Strimmer, 2008). We considered transcripts differentially expressed (DE) if the adjusted p-value for an effect was below 0.05. To examine whether differential expression calls were influenced by transcript abundance, we compared mean and median counts of DE and non-DE genes using two sample t-tests and Wilcoxon rank sum tests. We also compared overall patterns of gene-wise variance between DE and non-DE groups with respect to either population or rearing effect using Siegel-Tukey tests and Kolmogorov-Smirnov tests. We performed GO term enrichment analysis for all sets of DE transcripts using annotation information for 'Biological Processes' in the topGO package in R (Alexa \& Rahnenfuhrer, 2016).

\section{Overlap in identity and expression direction of DE gene sets within lineages}

To determine if transcripts exhibiting expression plasticity in response to rearing with predators were more likely to also exhibit evolutionary divergence between high- and low-predation populations, we evaluated overlap in transcripts differentially expressed between populations and between rearing conditions as well as their directions of expression change. To test the hypothesis that initial plastic responses following colonization of low-predation environments might drive subsequent divergence, we specifically compared pattern 
of ancestral plasticity (i.e. high-predation fish reared with and without predators) to genetic divergence (high- versus low-predation fish reared in the novel predator-free environment) within each drainage. We used post-hoc tests of simple effects to identify DE genes in these comparisons and estimate log-fold gene expression changes. We then used log-fold expression changes to determine whether gene expression differences were in the same/concordant direction (i.e. upregulated in high-predation populations and in response to rearing with predator cues, or down-regulated in high-predation populations and in response to rearing with predator cues) or opposite/non-concordant direction (upregulated in high-predation populations but down-regulated in response to rearing with predators, or vice versa).

Within each drainage, we compared the overlap in genes with both statistically significant genetic and plastic expression changes. We compared the association between directions of plasticity and divergence (concordant vs. non-concordant) in (1) those genes with both significant ancestral plasticity and significant population differences, and (2) all transcripts with significant population differences in expression, including those without significant expression plasticity. The second comparison addressed the possibility that even subthreshold expression plasticity in response to rearing environment could influence patterns of expression divergence, especially given the high false negative rate in our conservative DE analyses. Because these comparisons rely on two comparisons to a single group (high predation fish reared without exposure to predator cues), the estimates of plasticity and divergence are dependent. We adapted a parametric bootstrap method (Efron \& Tibshirani, 1993) similar to the approach implemented by (W. C. Ho \& Zhang, 2019) but accounting for conditional probabilities. We tested whether the conditional probabilities of gene expression divergence differed for genes that exhibited ancestral plasticity and those that did not. We further tested whether conditional probabilities for upregulated or downregulated genes in the low predation population compared to the high predation population differed depending on the direction of plasticity. We calculated parametric bootstrap confidence intervals for these differences in conditional probabilities as detailed in the Supplemental Methods, with confidence intervals that do not include zero indicating association between divergence and plasticity.

\section{Analysis of evolution of expression plasticity}

To test for the evolution of plasticity in gene expression responses, we examined the subset of transcripts with significant interaction effects in our differential expression analysis (see above). We grouped genes with significant interaction effects into categories for the evolution of plasticity outlined by Renn and Schumer (2013) based on post hoc differences between rearing environments within a population $(\mathrm{p}<0.05)$ : (1) Assimilated: plasticity in the ancestral high-predation population but a loss of plasticity in the derived low-predation population; (2) Accommodated: a change in the degree, but not the direction, of plasticity in the derived as compared to the ancestral population; (3) Reversed: opposing directions of plasticity in high- versus low-predation populations; (4) Evolved plastic: no plasticity in the ancestral high-predation population but an emergence of plasticity in the derived low-predation population; and (5) Unclassified: genes that had a significant main interaction effect but no significant post hoc rearing differences and could therefore not be unambiguously classified into one of the other categories. All statistical tests and data visualization were performed in R (version 3.5.1; The R Foundation for Statistical Computing).

\section{Parallelism in DE gene identity and expression direction between lineages}

To ask whether parallel phenotypic changes across independent lineages rely on shared gene expression changes, we evaluated overlap in DE transcript identity and expression direction between the Aripo and Quare lineage datasets. We used chi-square tests of independence to test for greater than chance overlap between drainages in those gene sets differentially expressed based on rearing environment or population of origin within drainages, as well as to test for biases in concordant versus non-concordant expression between drainages. We performed two additional comparisons to address potential bias in our conclusions due to high false negative rates in gene expression data (Rice, Schork, \& Rao, 2008). First, we performed the same analysis as before at two less stringent $\mathrm{p}$-value cut-offs $(\mathrm{p}=0.1$ and 0.2$)$. Second, for comparisons of expression direction, we made an additional, more conservative comparison by including not only those genes differentially expressed in both drainages (i.e. the intersection of DE gene lists), but also those differentially 
expressed in eitherdrainage (i.e. the union of DE gene lists) in the analysis. We again used log-fold expression differences to call gene expression as being in the same/concordant or opposite/non-concordant direction between drainages.

We also performed one addition comparison to assess parallelism in a subset of the population DE genes likely to arise from selection rather than other evolutionary processes, such as drift. We identified to those genes mostly likely to have diverged by selection, rather than by drift, by calculating $\mathrm{P}_{\mathrm{ST}}$ (a measure of phenotypic divergence between populations) from phenotypic variance components and comparing it to published estimates of $\mathrm{F}_{\mathrm{ST}}$ (a measure of neutral genetic divergence between populations) for the focal populations, using the method of Leinonen, Cano, Mäkinen, \& Merilä (2006). For these calculations, we made the conservative assumption that heritability $\left(h^{2}\right)$ of transcript abundance $=0.5$ as we have done previously (Ghalambor et al., 2015), and obtained estimates of within and between population variance from general linear mixed models (see Supplemental Methods). We then restricted DE gene sets in both lineages to those in which $\mathrm{P}_{\mathrm{ST}}>\mathrm{F}_{\mathrm{ST}}$ and compared the number of overlapping genes and their relative expression direction using chi-square tests as above. We used $\mathrm{F}_{\mathrm{ST}}$ estimates for our focal populations (Aripo $=0.225$; Quare $=0.340$ ) reported by Willing et al. (2010).

\section{Results}

\section{Differential gene expression and GO term enrichment}

Before testing our specific hypotheses, we first summarized differential expression patterns in both datasets. In the Aripo drainage, 766 genes were differentially expressed (DE) between high- and low-predation populations, 1,118 genes were differentially expressed between pred- and pred+ fish, and 825 genes had interaction effects (Fig. 2; Table S2). DE genes were enriched for GO categories including DNA integration, immune function, and morphogenesis. In the Quare drainage, 3,831 genes were differentially expressed between highand low-predation populations, 791 genes were differentially expressed between pred- and pred + fish, and 586 genes had interaction effects (Fig. 2; Table S3). DE genes were enriched for GO categories including immune function, developmental processes, and neurogenesis. Complete results of GO enrichment analyses for population of origin, rearing, and interaction effects are in the Supplemental Materials (Tables S4 \& S5).

Given the marked difference in the total number of DE genes between datasets, we looked for evidence that this pattern might arise from technical variation or expression bias. While we cannot rule out some influence of technical variation, we found no evidence for differences in sequence quality, read alignment statistics, or variance in transcript abundance to suggest that variation in the number of DE transcripts between drainages was of technical origin. Nor did we find evidence that identification of DE transcripts was biased by transcript expression level: mean and median gene expression were significantly lower in DE (population, rearing, and/or interaction effects) as compared to non-DE transcripts in both the Aripo (Fig. S1; mean: $\mathrm{DE}=263$, non-DE $=344, \mathrm{p}<0.0001$; median: $\mathrm{DE}=65$, non-DE $=107, \mathrm{p}<0.0001$ ), and Quare (Fig. S1; mean: $\mathrm{DE}=264$, non- $\mathrm{DE}=334, \mathrm{p}<0.0001$; median: $\mathrm{DE}=86$, non- $\mathrm{DE}=101, \mathrm{p}<0.0001)$ datasets. This suggests that identification of DE transcripts was not simply a side effect of relatively greater expression of these transcripts.

\section{Expression plasticity biases the propensity for and direction of genetic expression divergence}

Evolutionary theory and empirical studies suggest that environmentally induced plasticity may facilitate adaptation to novel environments. To test this prediction, we first asked whether genes with significant ancestral expression plasticity were more likely to show genetic divergence in expression. To compare expression changes upon colonization of low-predation environments by high-predation fish to those present after generations of adaptation to low-predation environments, we examined the extent of overlap in gene sets differentially expressed between high-predation fish reared with and without predators and high- versus low-predation fish reared in a shared, predator-free environment. In both drainages, the DE genes with significant ancestral plasticity were more likely to show significant population divergence than expected by chance (Aripo: 264 transcripts, 95\% CI for differences in conditional probability of significant divergence for plastic genes compared to conditional probability of divergence for non-plastic genes: [0.125, 0.150]; Qua- 
re: 409 transcripts, 95\% CI: [0.211,0.239]), supporting an association between plastic responses and genetic divergence.

We next asked whether the directions of expression plasticity and genetic divergence were associated. We did so for those genes with both significant ancestral plasticity and population differences, as well as the larger set of all transcripts with significant population differences (i.e. including those without significant expression plasticity). We performed the second, more conservative comparison because we reasoned that even subthreshold expression plasticity could influence the direction of adaptive divergence. Within both drainages, we found a signature of non-adaptive plasticity associated with genetic expression divergence. Approximately $84 \%$ of DE genes exhibited population and rearing expression changes in non-concordant directions in the Aripo drainage (95\% CI for difference in probability of same versus opposite expression direction: [-0.564,-0.499]), and $68 \%$ of DE genes exhibiting non-concordant expression in the Quare drainage (95\% CI: [-0.520,-0.456]) (Fig. 3). Patterns were consistent in both drainages when we considered the larger set of all genes with significant population expression differences (Aripo: 80\% of genes with non-concordant expression, 95\% CI: [-0.811,-0.752]; Quare: $66 \%$ of genes with non-concordant expression, 95\% CI: [-0.803,$0.733])$.

\section{Evolution of expression plasticity}

We next explored the evolution of expression plasticity itself. We identified genes with evolved expression plasticity as those in which the direction and/or degree of plasticity depended on population of origin, i.e. those genes with significant population-by-rearing interaction effects. The number of genes with significant expression plasticity that evolved (i.e. significant rearing and interaction effects) was approximately a quarter to a third of the number of genes with significant expression plasticity that did not evolve (i.e. significant rearing effect but not interaction; 263/1,118 in Aripo, 286/791 in Quare) (Fig. 2).

To characterize how expression plasticity evolved, we further subdivided genes with significant population-byrearing interaction effects into one of five categories: assimilated, accommodated, reversed, evolved plastic, or unclassified (Fig. 4A; based on Renn and Schumer, 2013). We found many transcripts that exhibited evolution of plasticity, with all five categories represented in both datasets (Fig. 4B). In the Aripo drainage $263(32 \%)$ transcripts showed patterns of expression assimilation, $81(10 \%)$ transcripts showed patterns of expression accommodation, $113(14 \%)$ transcripts exhibited reversed plasticity, and $182(22 \%)$ transcripts evolved plasticity in the derived population. In the Quare drainage, $164(28 \%)$ transcripts showed patterns of expression assimilation, $110(19 \%)$ transcripts showed patterns of expression accommodation, $128(22 \%)$ transcripts exhibited reversed plasticity, and $95(16 \%)$ transcripts evolved plasticity in the derived population (Fig. 4B).

\section{Parallelism in gene expression patterns across drainages}

To test for shared mechanisms mediating parallel phenotypic adaptation across drainages, we asked whether differentially expressed genes within drainages were overlapping in identity and expression direction between drainages. Of the genes that diverged between high- and low-predation populations within a drainage (i.e., population main effect), 174 were overlapping between drainages (Table S6), more than expected by chance $\left(\chi^{2}=8.02, \mathrm{p}=0.0046\right)$. However, the direction of expression divergence among these overlapping genes was not associated across the two drainages $\left(\chi^{2}=0.16, \mathrm{p}=0.686\right)$ : 91 genes had concordant expression changes and 83 genes had non-concordant expression changes between drainages (Fig. 5). To control for the influence of false negatives on our conclusions, we repeated this comparison at less stringent significance cut-offs. At a $\mathrm{p}=0.1$, we found marginally significant overlap in gene identify ( 347 overlapping genes; $\chi^{2}=3.38, \mathrm{p}=0.06$ ), but no association in expression direction $\left(\chi^{2}=0.65, \mathrm{p}=0.42\right)$, and at $\mathrm{p}=0.2$ we found no significant overlap or association in expression direction. As a final, more conservative test, we compared the subset of genes with strong evidence for adaptive population divergence due to selection by restricting our comparison to population $\mathrm{DE}$ genes for which $\mathrm{P}_{\mathrm{ST}}>\mathrm{F}_{\mathrm{ST}}$. We found 534 genes (mean $\left.\mathrm{P}_{\mathrm{ST}}=0.38, \mathrm{~F}_{\mathrm{ST}}=0.225\right)$ in the Aripo and 1163 genes (mean $\mathrm{P}_{\mathrm{ST}}=0.496, \mathrm{~F}_{\mathrm{ST}}=0.340$ ) in the Quare drainage that met this criterion. Of these, 53 were overlapping between drainages, again more than expected by chance $\left(\chi^{2}=12.7, p=0.0004\right)$, 
but without any association in expression direction (28 genes had concordant expression changes and 25 genes had non-concordant expression changes between drainages). $\mathrm{P}_{\mathrm{ST}}$ values for all genes are in Table S8.

Of the genes that showed significant expression plasticity (i.e. main effect of rearing), 64 transcripts were overlapping between drainages (Table S7), more than expected by chance $\left(\chi^{2}=14.06, p=0.0001\right)$. Of these 43 had expression changes in the same direction and 21 had expression changes in opposite directions between drainages, a significant association between drainages in the direction of expression plasticity associated with rearing environment $\left(\chi^{2}=7.56, \mathrm{p}=0.006\right)$ (Fig. 5). At less stringent $\mathrm{p}$-value cut-offs for differential expression we found no more overlap in gene identity than expected by chance $\left(\mathrm{p}<0.1\right.$ : 108 overlapping genes; $\chi^{2}=0.05$, $\mathrm{p}=0.81 ; \mathrm{p}<0.2$ : 276 overlapping genes; $\left.\chi^{2}=1.36, \mathrm{p}=0.24\right)$.

\section{Discussion}

We examined transcriptomic differences associated with adaptive divergence and rearing environment across repeated, independent evolutionary lineages in guppies. Within lineages, we observed phenotypic plasticity in gene expression patterns as well as the evolution of gene expression plasticity itself. Plastic genes were more likely to exhibit population differences in expression, and these population differences were more likely to be in the opposite, rather than the same, direction as ancestral expression plasticity. Comparing across lineages, gene sets differentially expressed were largely non-overlapping (i.e. $>75 \%$ non-shared identity) both for genes that diverged between populations and for plastic genes. Nonetheless, the small number of DE genes that did overlap between drainages was more than expected by chance for both population divergence and plasticity. Taken together our findings suggest that a small number of core genes may be repeatedly targeted during colonization of low-predation environments, but that largely non-shared, alternative transcriptional solutions are associated with parallel phenotypic adaptation across lineages.

\section{Impacts of gene expression plasticity on expression evolution}

A growing number of studies are using transcriptomic and proteomic surveys to address the long-standing debate on the role of plasticity in evolution, with contrasting results favoring the alternative hypotheses that adaptive (Fraser et al., 2014; Gleason \& Burton, 2015; Li et al., 2018; Mäkinen, Papakostas, Vøllestad, Leder, \& Primmer, 2016; Scoville \& Pfrender, 2010; Shaw et al., 2014; Wang \& Althoff, 2019) versus nonadaptive (Dayan, Crawford, \& Oleksiak, 2015; Ghalambor et al., 2015; W. Ho \& Zhang, 2018; Pespeni et al., 2013; Schaum, Rost, Millar, \& Collins, 2013) plasticity facilitates adaptation. We recently proposed that non-adaptive plasticity dominates during the earliest stages of rapid evolution, and that adaptive plasticity may contribute to subsequent fine-tuning of phenotypes (Fischer et al., 2016a). In line with this prediction and our previous findings, we report here that the strong signature of non-adaptive plasticity in brain gene expression (89\% of transcripts) observed in guppy populations in the earliest stages of adaptation to lowpredation environments (Ghalambor et al., 2015) is present but weaker in long-established low-predation populations. Furthermore, within our data set, this signature of non-adaptive plasticity was more apparent in the more recently diverged Aripo lineage (80\% of genes) as compared to the older Quare lineage (65\% of genes). Our findings highlight the need to explicitly consider how plasticity relates to divergence throughout successive stages of adaptation in order to build a more holistic understanding of the role of plasticity in adaptation.

\section{Evolution of plasticity in gene expression}

Diverse transcriptional patterns could accompany evolved differences in behavioral plasticity (Renn \& Schumer, 2013), but relevant data characterizing the evolution of gene expression plasticity in the brain has been lacking. In both lineages, we found approximately one third magnitude evolution of plasticity as we did conserved plasticity. As was observed for transcriptomic evolution of gill tissue in stickleback fish (Gibbons, Metzger, Healy, \& Schulte, 2017), plasticity evolution showed no consistent pattern, with genes gaining, losing, and switching the direction of expression plasticity between ancestral and derived populations in both lineages. The lack of consistency in these patterns and the dearth of studies that have characterized the evolution of gene expression plasticity make these patterns difficult to interpret at present. Some of the diversity is likely associated with adaptive phenotypic divergence between high- and low-predation populations, in which 
initially plastic behavioral shifts may become fixed, eliminated, or altered over time. For example, evolution in gene expression plasticity could reflect the gains, losses, and switches in plasticity of different behaviors in these populations (Fischer et al., 2016b). Alternatively, compensatory and homeostatic mechanisms could promote diversity among plastic responses in gene expression without altering higher-level phenotypic traits such as morphology and behavior (Badyaev, 2018; Fischer, Ghalambor, \& Hoke, 2016a; Renn \& Schumer, 2013). Finally, because fish in low-predation habitats experience relaxed selection on predator-induced plasticity in conjunction with low effective populations sizes, some of the evolution of transcriptional plasticity we report likely arose as a product of genetic drift, rather than selection for altered plastic responses (Lynch, 2007). Documenting the evolution of expression plasticity is an important first step, and additional studies are needed to understand the ubiquity and evolutionary sources of these patterns.

\section{Transcriptomic signatures of population divergence in two independent evolutionary lineages}

We identified many genes differentially expressed between high- and low-predation populations in each river drainage. The absolute number of population DE transcripts was smaller in the Aripo drainage as compared to the Quare drainage, but the number of developmental and interaction differences was greater in the Aripo drainage. The Quare dataset was 1.5X larger than the Aripo dataset (40 versus 60 total samples) and the identification of a greater number of DE genes in the Quare dataset is therefore likely in part related to greater statistical power. This difference may also be biological in origin, as high- and low-predation populations in the Quare drainage show greater genetic divergence than those in the Aripo drainage (Willing et al., 2010). Indeed, we note that the larger number of genes in the Quare dataset come from a large difference the number of genes with population, but not rearing or interaction, effects. In sum, we suggest that the difference in the number of differentially expressed transcripts between datasets is likely a combination of greater statistical power in the larger Quare dataset and the greater degree of genetic divergence in the Quare as compared to the Aripo river drainage (Willing et al., 2010).

When we compared DE gene sets between drainages, we found a small, but significant, number of population and rearing DE genes shared across lineages. However, the majority of DE genes were non-shared across drainages for both population (Aripo: 78\%; Quare: 96\%) and rearing (Aripo: 94\%; Quare: 92\%) effects. Only rearing genes showed a significant association in expression direction, with $67 \%$ of genes concordantly differentially expressed between lineages. In contrast, only $52 \%$ of the genes that diverged when low-predation fish colonized high-predation habitats in both drainages were concordantly differentially expressed. We suggest that these patterns across drainages point to a small number of core genes that exhibit predictable, plastic expression responses upon colonization of low-predation environments, but that lineage-specific selection pressures, differences in genetic background, non-adaptive processes (e.g. drift, inbreeding, founder effects), and alternative compensatory gene expression responses give rise to largely non-overlapping, non-concordant expression differences associated with parallel phenotypic adaptation across drainages.

A previous study in guppies performed a similar comparison of gene expression changes associated with adaptation to low-predation environments (Ghalambor et al., 2015) and found a strong signal of concordant differential expression in genes differentially expressed based on population of origin. Whereas the present study compared long-term natural population divergence across drainages, Ghalambor et al. (2015) characterized early stages of adaptation of experimentally introduced low-predations populations derived from founders from a single high-predation source population within the same drainage. These contrasting findings in comparisons of population pairs within the same drainage versus across drainages highlight the impacts of standing genetic variation within the source population on mechanisms of divergence (Feiner, Rago, While, \& Uller, 2017; Thompson, Osmond, \& Schluter, 2019), particularly at early stages of evolution (Barrett \& Schluter, 2008): while alternative transcriptional 'solutions' are possible, shared genetic background appears to bias evolutionary outcomes toward shared patterns.

Both adaptive and non-adaptive processes may contribute to the combination of shared and distinct transcriptional mechanisms we find associated with parallel, adaptive life-history, morphological, and behavioral phenotypes across lineages in guppies. First, as described above, differences in standing genetic variation likely influence which mechanisms are available to selection in response to common environmental conditions 
in different drainages (Barrett \& Schluter, 2008; Thompson et al., 2019). Second, low-predation populations are typically established by a very small number of individuals (Barson et al., 2009; Fraser et al., 2015; Willing et al., 2010), making them susceptible to the unpredictable, non-adaptive influences of founder's effects, genetic drift, and/or inbreeding on gene expression divergence - although we note that we found no more evidence for shared mechanisms among genes most likely under selection than among all diverged genes (i.e. genes with $\mathrm{P}_{\mathrm{ST}}>\mathrm{F}_{\mathrm{ST}}$ ). Third, the large number of significantly evolved genes that did not overlap between drainages may also represent adaptive responses to drainage- or site-specific environmental factors other than predation (Fitzpatrick, Torres-Dowdall, Reznick, Ghalambor, \& Chris Funk, 2014; Zandonà et al., 2011). Finally, alternative compensatory or homeostatic gene expression responses may arise in response to any of the above factors, leading to alternative transcriptional configurations associated with similar higher-level phenotypes. In other words, genetic similarity among ancestral populations may channel lowpredation populations within the same drainage toward shared transcriptional solutions (as in Ghalambor et al. 2015), while differences in standing genetic variation, drainage-specific environmental conditions, founder effects, and alternative compensatory changes could result in distinct mechanistic paths to arrive at shared organism-level phenotypes. We cannot definitely distinguish causal from non-adaptive and compensatory gene expression differences under these scenarios - indeed, it is likely a combination of these factors that contribute to distinct transcriptional patterns associated with parallel adaptation. Nonetheless, in either case, alternative transcriptional patterns suggest that mechanistic flexibility and 'many-to-one' mapping of gene expression to organism level phenotypes may facilitate adaptation.

\section{Conclusions}

We assessed the extent to which adaptation to common environments targets predictable changes in transcript abundance across independent evolutionary events. Within lineages, genes with greater expression plasticity were more likely to diverge in expression between populations, but in the direction opposite of plastic changes. Despite a small number of differentially expressed genes shared across drainages, parallel adaptation to lowpredation environments in independent lineages was associated with divergence in largely non-overlapping transcripts. While identification of shared genes is generally used as the starting point for work exploring mechanisms of parallel adaptation - and our work here indeed suggests that a small number of core genes may indeed be critical during the earliest stages of adaptation - we propose that parallel evolutionary transitions are not limited to a small set of possible transcriptional mechanisms in guppies. Instead, our results highlight the potential for alternative transcriptional solutions associated with parallel, adaptive trait evolution even within a single species. Transcriptional network versatility, in which diverse alterative network configurations can produce common network outputs and behavioral phenotypes, may allow underlying networks to simultaneously accommodate the influences of selection, drift, and genetic background and thereby facilitate evolution in a species known for rapid adaptation to novel environments.

Acknowledgements : We thank the members of the Colorado State University Guppy Group for fish care, especially Sarah E Westrick and Kimberly E Dolphin for help with tissue collection. We thank Laura R Stein for assistance with tissue processing and Cameron K Ghalambor for fruitful discussions and comments on earlier versions of the manuscript. All high-powered computing was performed on the Odyssey computing cluster supported by the FAS Science Division Research Computing Group at Harvard University. This work was supported by the National Science Foundation DDIG-1311680 (to EKF), RCN IOS-1256839 (to EKF), IOS-1354755 (to KLH), IOS-1354775 (to KAH), IOS-0934451 (to KAH), DEB-0846175 (to CKG), IIS-1545994 (to WZ), and US Department of Energy DE-SC0018344 (to WZ).

Data Accessibility : Raw sequencing reads will be made available through the NCBI SRA repository upon publication. $\mathrm{R}$ code for statistical analyses will be made available on GitHub upon publication.

Author contributions : EKF and KLH conceived of the study; EKF collected samples and performed molecular work; EKF and KAH performed gene expression mapping, transcript abundance estimation, and preliminary differential expression analyses; YS and WZ devised and performed statistical analyses with input from EKF and KLH; EKF and KLH wrote the manuscript with contributions from all authors. All authors gave final approval for publication. 


\section{References}

Abouheif, E., \& Wray, G. (2002). Evolution of the gene network underlying wing polymorphism in ants. Science, 297 (2002), 249-252. doi: 10.1126/science.1071468

Alberch, P. (1991). From genes to phenotype: dynamical systems and evolvability. Genetica , 84 , 5-11. doi: 10.1007/BF00123979

Alexa, A., \& Rahnenfuhrer, J. (2016). topGO: Enrichment Analysis for Gene Ontoloty . R package version 2.30 .1 .

Badyaev, A. V. (2018). Evoulutionary transitions in controls reconcile adaptation with continuity of evolution. Seminars in Cell and Developmental Biology , 88 , 36-45. doi: 10.1016/j.semcdb.2018.05.014

Badyaev, A. V, \& Morrison, E. S. (2018). Emergent buffering balances evolvability and robustness in the evolution of phenotypic flexibility.Evolution , 72 (3), 647-662. doi: 10.1111/evo.13441

Baldwin, J. M. (1896). A New Factor in Evolution. The American Naturalist, 30 , 441-451; 536-553.

Barrett, R. D. H., \& Schluter, D. (2008). Adaptation from standing genetic variation. Trends in Ecology and Evolution , 23 (1), 38-44. doi: 10.1016/j.tree.2007.09.008

Barson, N. J., Cable, J., \& Van Oosterhout, C. (2009). Population genetic analysis of microsatellite variation of guppies (Poecilia reticulata) in Trinidad and Tobago: Evidence for a dynamic source-sink metapopulation structure, founder events and population bottlenecks. Journal of Evolutionary Biology , 22 (3), 485-497. doi: 10.1111/j.1420-9101.2008.01675.x

Conover, D. O., Duffy, T. A., \& Hice, L. A. (2009). The covariance between genetic and environmental influences across ecological gradients: Reassessing the evolutionary significance of countergradient and cogradient variation. Annals of the New York Academy of Sciences , 1168 , 100-129. doi: 10.1111/j.17496632.2009.04575.x

Crawford, D. L., \& Oleksiak, M. F. (2007). The biological importance of measuring individual variation. Journal of Experimental Biology ,210 (9), 1613-1621. doi: 10.1242/jeb.005454

Dayan, D. I., Crawford, D. L., \& Oleksiak, M. F. (2015). Phenotypic plasticity in gene expression contributes to divergence of locally adapted populations of Fundulus heteroclitus . Molecular Ecology , 24 (13), 33453359. doi: $10.1111 /$ mec. 13188

Dobin, A., Davis, C. A., Schlesinger, F., Drenkow, J., Zaleski, C., Jha, S., .. Gingeras, T. R. (2013). STAR: Ultrafast universal RNA-seq aligner. Bioinformatics , 29 (1), 15-21. doi: 10.1093/bioinformatics/bts635

Draghi, J. A., \& Whitlock, M. C. (2012). Phenotypic plasticity facilitates mutational variance, genetic variance, and evolvability along the major axis of environmental variation. Evolution ,66 (9), 2891-2902. doi: 10.1111/j.1558-5646.2012.01649.x

Drion, G., O'Leary, T., \& Marder, E. (2015). Ion channel degeneracy enables robust and tunable neuronal Firing rates. PNAS . doi: 10.1073/pnas.1516400112

Efron, B., \& Tibshirani, R. J. (1993). An introduction to the bootstrap. Monographs on Statistics and Applied Probability, No. 57. Chapman and Hall, London, 436 p. In Monographs on Statistics and Applied Probability .

Elvidge, C. K., Ramnarine, I., \& Brown, G. E. (2014). Compensatory foraging in Trinidadian guppies: Effects of acute and chronic predation threats. Current Zoology , 60 (3), 323-332. doi: 10.1093/czoolo/60.3.323

Endler, J. A. (1995). Multiple-trait coevolution and environmental gradients in guppies. Trends in Ecology E Evolution ,10 (1), 22-29. doi: 10.1016/S0169-5347(00)88956-9 
Espinosa-Soto, C., Martin, O. C., \& Wagner, A. (2011). Phenotypic plasticity can facilitate adaptive evolution in gene regulatory circuits. BMC Evolutionary Biology , 11 (5), 1-14. doi: 10.1186/1471-2148-11-5

Fajen, A. V. A., \& Breden, F. (1992). Mitochondrial DNA sequence variation among natural populations of the Trinidad guppy,Poecilia reticulata . Evolution , 46 (5), 1457-1465.

Feiner, N., Rago, A., While, G. M., \& Uller, T. (2017). Signatures of selection in embryonic transcriptomes of lizards adapting in parallel to cool climate. Evolution, 72 (1), 67-81. doi: 10.1111/evo.13397

[dataset] Fischer, E. K., Hughes, K. A., Hoke, K. L. (2020). NCBI Sequence Read Archive. SUB6850296.

Fischer, E. K., Ghalambor, C. K., \& Hoke, K. L. (2016a). Can a Network Approach Resolve How Adaptive vs Nonadaptive Plasticity Impacts Evolutionary Trajectories? Integrative and Comparative Biology ,56 (5), 877-888. doi: 10.1093/icb/icw087

Fischer, E. K., Ghalambor, C. K., \& Hoke, K. L. (2016b). Plasticity and evolution in correlated suites of traits. Journal of Evolutionary Biology , 29 (5), 991-1002. doi: 10.1111/jeb.12839

Fischer, E. K., Harris, R. M., Hofmann, H. A., \& Hoke, K. L. (2014). Predator exposure alters stress physiology in guppies across timescales. Hormones and Behavior , 65 (2), 165-172. doi: 10.1016/j.yhbeh.2013.12.010

Fischer, E. K., Soares, D., Archer, K. R., Ghalambor, C. K., \& Hoke, K. L. (2013). Genetically and environmentally mediated divergence in lateral line morphology in the Trinidadian guppy (Poecilia reticulata). Journal of Experimental Biology , 216 (16), 3132-3142. doi: 10.1242/jeb.081349

Fitzpatrick, S. W., Torres-Dowdall, J., Reznick, D. N., Ghalambor, C. K., \& Chris Funk, W. (2014). Parallelism Isn't Perfect: Could Disease and Flooding Drive a Life-History Anomaly in Trinidadian Guppies? The American Naturalist , 183 (2), 290-300. doi: 10.1086/674611

Fraser, B. A., Janowitz, I., Thairu, M., Travis, J., Hughes, K. A., Fraser, B. A., .. Hughes, K. A. (2014). Phenotypic and genomic plasticity of alternative male reproductive tactics in sailfin mollies.Proceedings of the Royal Society B: Biological Sciences ,281, 20132310.

Fraser, B. A., Künstner, A., Reznick, D. N., Dreyer, C., \& Weigel, D. (2015). Population genomics of natural and experimental populations of guppies (Poecilia reticulata). Molecular Ecology ,24 (2), 389-408. doi: $10.1111 /$ mec. 13022

Ghalambor, C. K., Hoke, K. L., Ruell, E. W., Fischer, E. K., Reznick, D. N., \& Hughes, K. A. (2015). Nonadaptive plasticity potentiates rapid adaptive evolution of gene expression in nature. Nature ,525 (7569), 372-375. doi: 10.1038/nature15256

Ghalambor, C. K., McKay, J. K., Carroll, S. P., \& Reznick, D. N. (2007). Adaptive versus non-adaptive phenotypic plasticity and the potential for contemporary adaptation in new environments. Functional Ecology , 21 (3), 394-407. doi: 10.1111/j.1365-2435.2007.01283.x

Ghalambor, C. K., Reznick, D. N., \& Walker, J. A. (2004). Constraints on adaptive evolution: The functional trade-off between reproduction and fast-start swimming performance in the Trinidadian guppy (Poecilia reticulata ). American Naturalist , 164 (1), 38-50. doi: 10.1086/421412

Gibbons, T. C., Metzger, D. C., Healy, T. M., \& Schulte, P. M. (2017). Gene expression plasticity in response to salinity acclimation in threespine stickleback ecotypes from different salinity habitats. Molecular Ecology , 26 , 2711-2725. doi: 10.1111/mec.14065

Gilliam, J. F., Fraser, D. F., \& Alkins-Koo, M. (1993). Structure of a Tropical Stream Fish Community : A Role for Biotic Interactions.Ecology, 74 (6), 1856-1870.

Gleason, L. U., \& Burton, R. S. (2015). RNA-seq reveals regional differences in transcriptome response to heat stress in the marine snailChlorostoma funebralis . Molecular Ecology , 24 (3), 610-627. doi: $10.1111 /$ mec. 13047 
Gompel, N., \& Prud'homme, B. (2009). The causes of repeated genetic evolution. Developmental Biology , 332 (1), 36-47. doi: 10.1016/j.ydbio.2009.04.040

Grashow, R., Brookings, T., \& Marder, E. (2009). Reliable neuromodulation from circuits with variable underlying structure.PNAS , 106 (28), 11742-11746. doi: 10.1073/pnas.0905614106

Grether, G. F. (2005). Environmental Change, Phenotypic Plasticity, and Genetic Compensation. The American Naturalist , 166 (4), E115-E123. doi: 10.1086/432023

Handelsman, C. A., Ruell, E. W., Torres-Dowdall, J., \& Ghalambor, C. K. (2014). Phenotypic Plasticity Changes Correlations of Traits Following Experimental Introductions of Trinidadian Guppies (Poecilia reticulata ). Integrative and Comparative Biology , 54 (5), 794-804. doi: 10.1093/icb/icu112

Handelsman, Corey A., Broder, E. D., Dalton, C. M., Ruell, E. W., Myrick, C. A., Reznick, D. N., \& Ghalambor, C. K. (2013). Predator-induced phenotypic plasticity in metabolism and rate of growth: Rapid adaptation to a novel environment. Integrative and Comparative Biology , 53 (6), 975-988. doi: 10.1093/icb/ict057

Harris, S., Ramnarine, I. W., Smith, H. G., \& Pettersson, L. B. (2010). Picking personalities apart: Estimating the influence of predation, sex and body size on boldness in the guppy Poecilia reticulata. Oikos . doi: $10.1111 /$ j.1600-0706.2010.18028.x

Haskins, C., Haskins, E., McLaughlin, J., \& Hewitt, R. (1961). Polymorphism and population structure in Lebistes reticulatus, an ecological study. In F. Blair (Ed.), Vertebrate speciation (pp. 320-395). Austin: University of Austin Press.

Ho, W. C., \& Zhang, J. (2019). Genetic Gene Expression Changes during Environmental Adaptations Tend to Reverse Plastic Changes Even after the Correction for Statistical Nonindependence. Molecular Biology and Evolution , 36 (3), 604-612. doi: 10.1093/molbev/msz002

Ho, W., \& Zhang, J. (2018). Evolutionary adaptations to new environments generally reverse plastic phenotypic changes. Nature Communications , 9 (350), 1-11. doi: 10.1038/s41467-017-02724-5

Huizinga, M., Ghalambor, C. K., \& Reznick, D. N. (2009). The genetic and environmental basis of adaptive differences in shoaling behaviour among populations of Trinidadian guppies, Poecilia reticulata . Journal of Evolutionary Biology , 22 (9), 1860-1866. doi: 10.1111/j.1420-9101.2009.01799.x

Insel, T. R., \& Young, L. J. (2000). Neuropeptides and the evolution of social behavior. Current Opinion in Neurobiology , 10 (6), 784-789. doi: 10.1016/S0959-4388(00)00146-X

Lande, R. (2009). Adaptation to an extraordinary environment by evolution of phenotypic plasticity and genetic assimilation. Journal of Evolutionary Biology , 22 (7), 1435-1446. doi: 10.1111/j.14209101.2009.01754.x

Lehmann, E. L., \& Romano, J. P. (2005). Testing Statistical Hypotheses (Third Edit). New York: Springer.

Leinonen, T., Cano, J. M., Makinen, H., \& Merila, J. (2006). Contrasting patterns of body shape and neutral genetic divergence in marine and lake populations of threespine sticklebacks. Journal of Evolutionary Biology , 19 , 1803-1812. doi: 10.1111/j.1420-9101.2006.01182.x

Li, L., Li, A., Song, K., Meng, J., Guo, X., Li, S., . . Xu, F. (2018). Divergence and plasticity shape adaptive potential of the Pacific oyster. Nature Ecology Evolution , 2 , 1751-1760. doi: 10.1038/s41559-018-0668-2

Losos, J. B. (2011). Convergence, adaptation, and constraint.Evolution , 65 (7), 1827-1840. doi: $10.1111 / \mathrm{j} .1558-5646.2011 .01289 . \mathrm{x}$

Love, M. I., Huber, W., \& Anders, S. (2014). Moderated estimation of fold change and dispersion for RNA-seq data with DESeq2. Genome Biology , 15 (12), 1-21. doi: 10.1186/s13059-014-0550-8

Lynch, M. (2007). The frailty of adaptive hypotheses for the origins of organismal complexity. PNAS , 104 , 8597-8604. doi: 10.17226/11790 
Magurran, A. E. (2005). Evolutionary ecology: the Trinidadian guppy. InOxford Series in Ecology and Evolution (Vol. 19). doi: 10.1093/acprof

Magurran, A. E., \& Seghers, B. H. (1990a). Population differences in predator recognition and attack cone avoidance in the guppyPoecilia reticulata . Animal Behaviour , 40 (3), 443-452. doi: 10.1016/S00033472(05)80524-X

Magurran, A. E., \& Seghers, B. H. (1990b). Population Differences in the Schooling Behaviour of Newborn Guppies, Poecilia reticulata.Ethology , 84 , 334-342. doi: 10.1111/j.1439-0310.1990.tb00807.x

Magurran, A. E., \& Seghers, B. H. (1991). Variation in Schooling and Aggression Amongst Guppy (Poecilia Reticulata ) Populations in Trinidad. Behaviour , 188 (3-4), 214-234. doi: 10.1163/156853991X00292

Magurran, A. E., \& Seghers, B. H. (1994). Predator Inspection Behaviour Covaries with Schooling Tendency Amongst Wild Guppy, Poecilia Reticulata, Populations in Trinidad. Behaviour ,128 (1-2), 121-134. doi: 10.1163/156853994X00073

Makinen, H., Papakostas, S., Vollestad, L. A., Leder, E. H., \& Primmer, C. R. (2016). Plastic and evolutionary gene expression responses are correlated in European grayling (Thymallus thymallus ) subpopulations adapted to different thermal environments. Journal of Heredity , $10^{77}$ (1), 82-89. doi: 10.1093/jhered/esv069

Manceau, M., Domingues, V. S., Linnen, C. R., Rosenblum, E. B., \& Hoekstra, H. E. (2010). Convergence in pigmentation at multiple levels: mutations, genes and function. Philosophical Transactions of the Royal Society B: Biological Sciences , 365 (1552), 2439-2450. doi: 10.1098/rstb.2010.0104

Mandic, M., Ramon, M. L., Gerstein, A. C., Gracey, A. Y., \& Richards, J. G. (2018). Variable gene transcription underlies phenotypic convergence of hypoxia tolerance in sculpins. BMC Evolutionary Biology , 18 , 163. doi: 10.1186/s12862-018-1275-1

Marder, E., \& Goaillard, J. M. (2006). Variability, compensation and homeostasis in neuron and network function. Nature Reviews Neuroscience , 7 , 563-574. doi: 10.1038/nrn1949

Pankey, M. S., Minin, V. N., Imholte, G. C., Suchard, M. A., \& Oakley, T. H. (2014). Predictable transcriptome evolution in the convergent and complex bioluminescent organs of squid. PNAS, 111 (44), E4736-E4742. doi: 10.1073/pnas.1416574111

Pespeni, M. H., Sanford, E., Gaylord, B., Hill, T. M., Hosfelt, J. D., Jaris, H. K., .. Palumbi, S. R. (2013). Evolutionary change during experimental ocean acidification. PNAS , 110 (17), 6937-6942. doi: $10.1073 /$ pnas. 1220673110

Pigliucci, M. (2010). Genotype-phenotype mapping and the end of the "genes as blueprint" metaphor. Philosophical Transactions of the Royal Society B: Biological Sciences , 365 , 557-566. doi: 10.1098/rstb.2009.0241

Renn, S. C. P., \& Schumer, M. E. (2013). Genetic accommodation and behavioural evolution : insights from genomic studies. Animal Behaviour , 85 (5), 1012-1022. doi: 10.1016/j.anbehav.2013.02.012

Reznick, D. A., Bryga, H., \& Endler, J. A. (1990). Experimentally induced life-history evolution in a natural population. Nature ,346 (6282), 357-359. doi: 10.1038/346357a0

Reznick, D., Butler IV, M. J., \& Rodd, H. (2001). Life-History Evolution in Guppies. VII. The Comparative Ecology of High- and Low-Predation Environments. The American Naturalist ,157 (2), 126-140. doi: $10.1086 / 318627$

Reznick, D. N. (1997). Life history evolution in guppies (Poecilia reticulata): Guppies as a model for studying the evolutionary biology of aging. Experimental Gerontology , 32 (3), 245-258. doi: 10.1016/S0531$5565(96) 00129-5$ 
Reznick, D. N., \& Bryga, H. (1987). Life-History Evolution in Guppies (Poecilia reticulata ): 1. Phenotypic and Genetic Changes in an Introduction Experiment. Evolution , 41 (6), 1370. doi: 10.2307/2409101

Rice, T. K., Schork, N. J., \& Rao, D. C. (2008). Methods for Handling Multiple Testing. Advances in Genetics , 60 , 293-308. doi: 10.1016/S0065-2660(07)00412-9

Rosenblum, E. B., Rompler, H., Schoneberg, T., \& Hoekstra, H. E. (2010). Molecular and functional basis of phenotypic convergence in white lizards at White Sands. PNAS , 107 (5), 2113-2117. doi: 10.1073/pnas.0911042107

Ruell, E. W., Handelsman, C. A., Hawkins, C. L., Sofaer, H. R., Ghalambor, C. K., \& Angeloni, L. (2013). Fear, food and sexual ornamentation: plasticity of colour development in Trinidadian guppies.Proceedings of the Royal Society B: Biological Sciences ,280 (1758), 20122019-20122019. doi: 10.1098/rspb.2012.2019

Schaum, E., Rost, B., Millar, A. J., \& Collins, S. (2013). Variation in plastic responses of a globally distributed picoplankton species to ocean acidification. Nature Climate Change , 3 (3), 298-302. doi: 10.1038/nclimate1774

Scoville, A. G., \& Pfrender, M. E. (2010). Phenotypic plasticity facilitates recurrent rapid adaptation to introduced predators. PNAS , 107 (9), 4260-4263. doi: 10.1073/pnas.0912748107

Shaw, J. R., Hampton, T. H., King, B. L., Whitehead, A., Galvez, F., Gross, R. H., ... Stanton, B. A. (2014). Natural selection canalizes expression variation of environmentally induced plasticity-enabling genes. Molecular Biology and Evolution ,31 (11), 3002-3015. doi: 10.1093/molbev/msu241

Siegal, M. L., \& Leu, J.-Y. (2014). On the Nature and Evolutionary Impact of Phenotypic Robustness Mechanisms. Annual Review of Ecology, Evolution, and Systematics , 45 (1), 495-517. doi: 10.1146/annurevecolsys-120213-091705

Song, L., \& Florea, L. (2015). Rcorrector: Efficient and accurate error correction for Illumina RNA-seq reads. GigaScience , 4 (1), 1-8. doi: 10.1186/s13742-015-0089-y

Stern, D. L., \& Orgogozo, V. (2008). The loci of evolution: How predictable is genetic evolution? Evolution ,62 (9), 2155-2177. doi: 10.1111/j.1558-5646.2008.00450.x

Storey, J. D. (2002). A direct approach to false discovery rates. Journal of the Royal Statistical Society. Series B: Statistical Methodology , 64 , 479-498. doi: 10.1111/1467-9868.00346

Strimmer, K. (2008). fdrtool: A versatile R package for estimating local and tail area-based false discovery rates. Bioinformatics ,24 (12), 1461-1462. doi: 10.1093/bioinformatics/btn209

Thompson, K. A., Osmond, M. M., \& Schluter, D. (2019). Parallel genetic evolution and speciation from standing variation. Evolution Letters , 3 (2), 129-141. doi: 10.1002/evl3.106

Torres-Dowdal, J., Handelsman, C. a, Reznick, D. N., \& Ghalambor, C. K. (2012). Local adaptation and the evolution of of phenotypic plasticity in Trinidadian guppies (Poecilia reticulata). Evolution ,66 (11), 3432-3443. doi: 10.5061/dryad.84gf5

Torres Dowdall, J., Handelsman, C. A., Ruell, E. W., Auer, S. K., Reznick, D. N., \& Ghalambor, C. K. (2012). Fine-scale local adaptation in life histories along a continuous environmental gradient in Trinidadian guppies. Functional Ecology , 26 (3), 616-627. doi: 10.1111/j.1365-2435.2012.01980.x

Velotta, J. P., \& Cheviron, Z. A. (2018). Remodeling Ancestral Phenotypic Plasticity in Local Adaptation : A New Framework to Explore the Role of Genetic Compensation in the Evolution of Homeostasis.Integrative and Comparative Biology , 58 (6), 1098-1110. doi: 10.1093/icb/icy117

Wagner, A. (2011). The origins of evolutionary innovations: a theory of transformative change in living systems. Oxford: Oxford University Press. 
Wang, S. P., \& Althoff, D. M. (2019). Phenotypic plasticity facilitates initial colonization of a novel environment. Evolution ,73 (2), 303-316. doi: 10.1111/evo.13676

West-Eberhard, M. J. (2003). Developmental plasticity and evolution . doi: 10.2002/ajpa.20219

Willing, E. M., Bentzen, P., Van Oosterhout, C., Hoffmann, M., Cable, J., Breden, F., .. Dreyer, C. (2010). Genome-wide single nucleotide polymorphisms reveal population history and adaptive divergence in wild guppies. Molecular Ecology , 19 (5), 968-984. doi: 10.1111/j.1365-294X.2010.04528.x

Yang, J. R., Maclean, C. J., Park, C., Zhao, H., \& Zhang, J. (2017). Intra and interspecific variations of gene expression levels in yeast are largely neutral. Molecular Biology and Evolution . doi: 10.1093/molbev/msx171

Zandona, E., Auer, S. K., Kilham, S. S., Howard, J. L., Lopez-Sepulcre, A., O'Connor, M. P., .. Reznick, D. N. (2011). Diet quality and prey selectivity correlate with life histories and predation regime in Trinidadian guppies. Functional Ecology , 25 (5), 964-973. doi: 10.1111/j.1365-2435.2011.01865.x

Figures 
A. natural populations
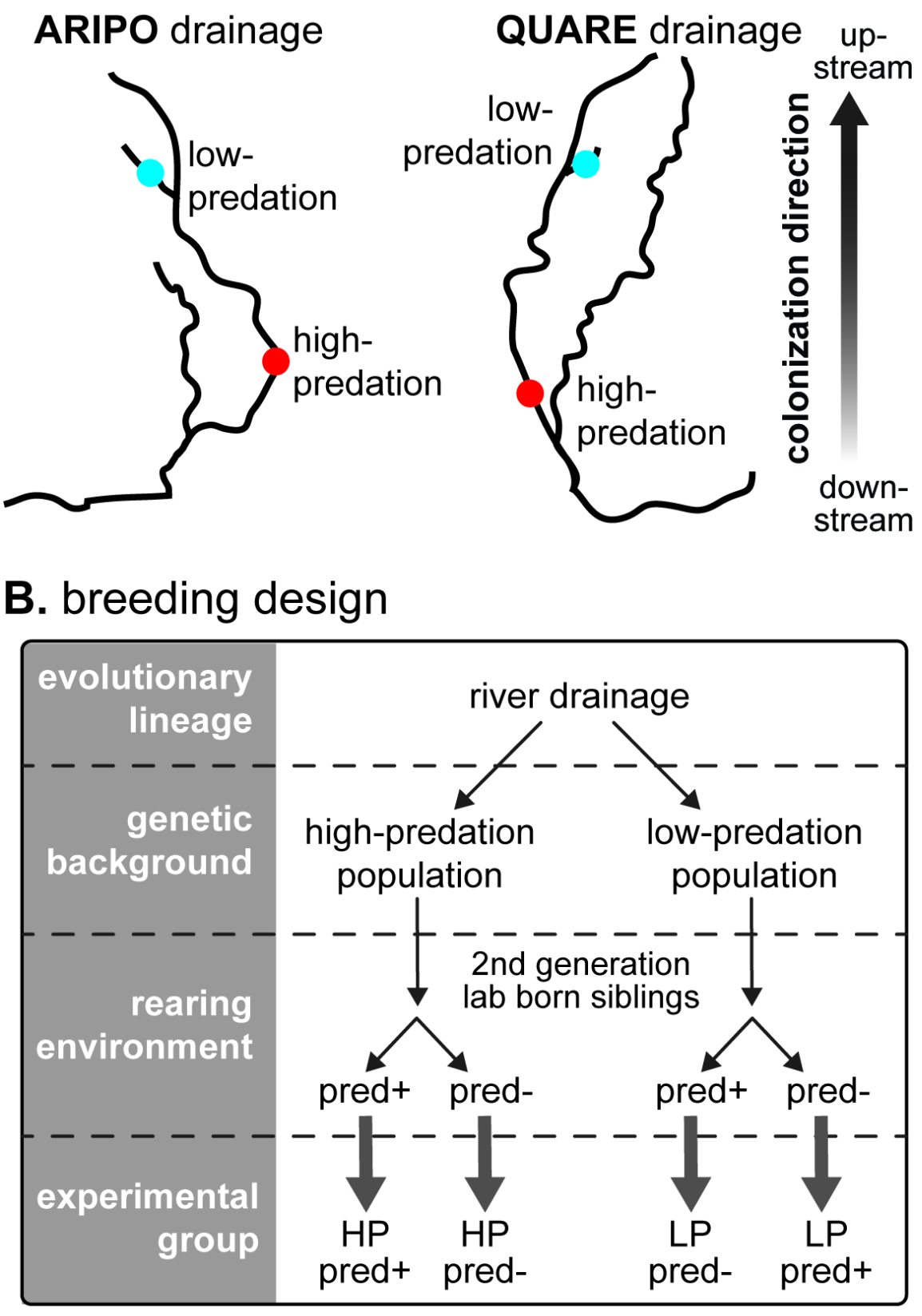

C. interpretation
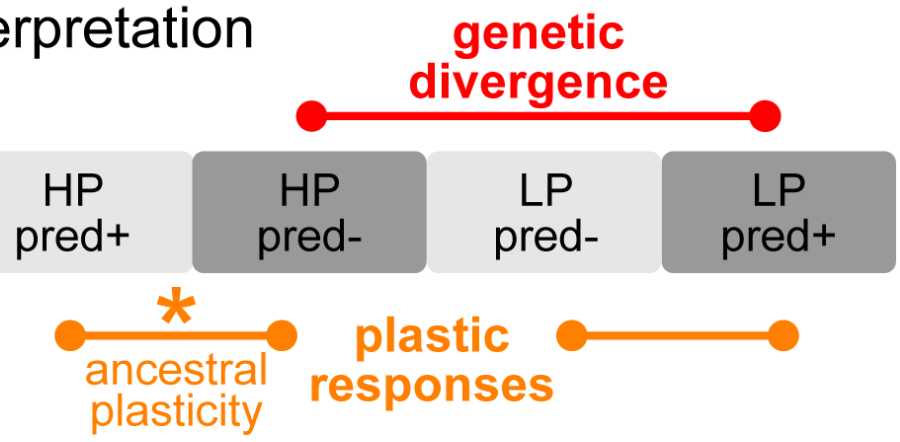
Figure 1. Overview of experimental design and interpretation. (A) Schematic shows Aripo and Quare river drainages in the Northern Range Mountains of Trinidad. We sampled population pairs from these two drainages, which represent distinct evolutionary lineages. (B) Wild-caught fish from each high- and low-predation population pair were reared to the second generation in the lab to control for maternal and environmental effects. At birth, second generation siblings from each population were split into rearing environments with (pred+) and without (pred-) predation cues. (C) This rearing design allows us to discern lineage effects (contrast between drainages), genetic divergence (contrast between different populations in the same environment), phenotypic plasticity (contrast between genetically similar fish in different rearing condition), and their interactions.

\section{ARIPO drainage}

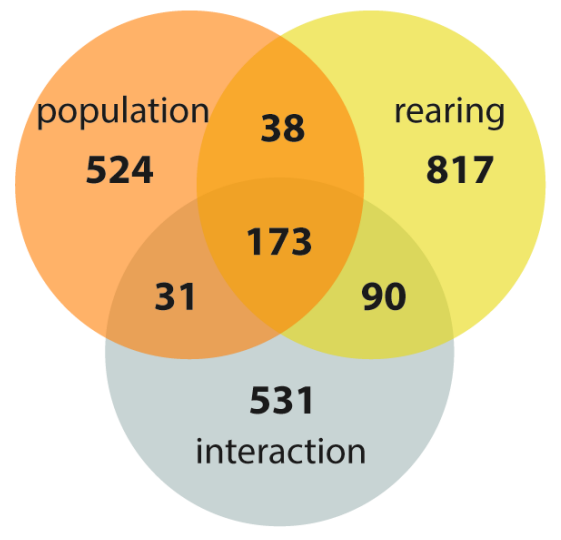

QUARE drainage

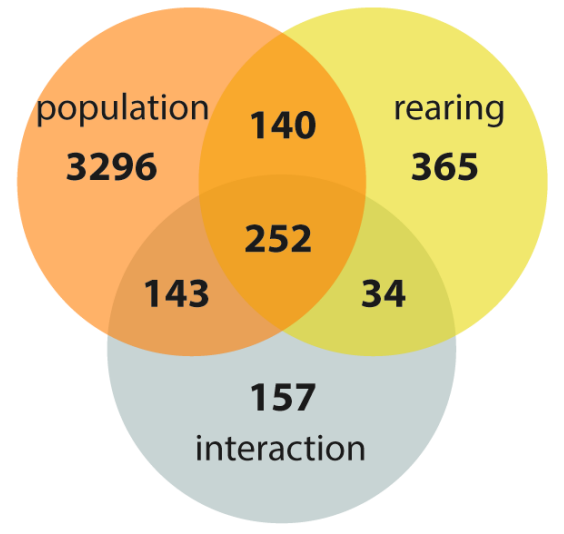

Figure 2 . Summary of differential expression analyses. We identified many transcripts in both the Aripo (left) and Quare (right) drainages whose expression levels differed based on evolutionary history of predation (population of origin; orange), developmental experience of predation (rearing; yellow), and their interaction (grey). 
ARIPO drainage

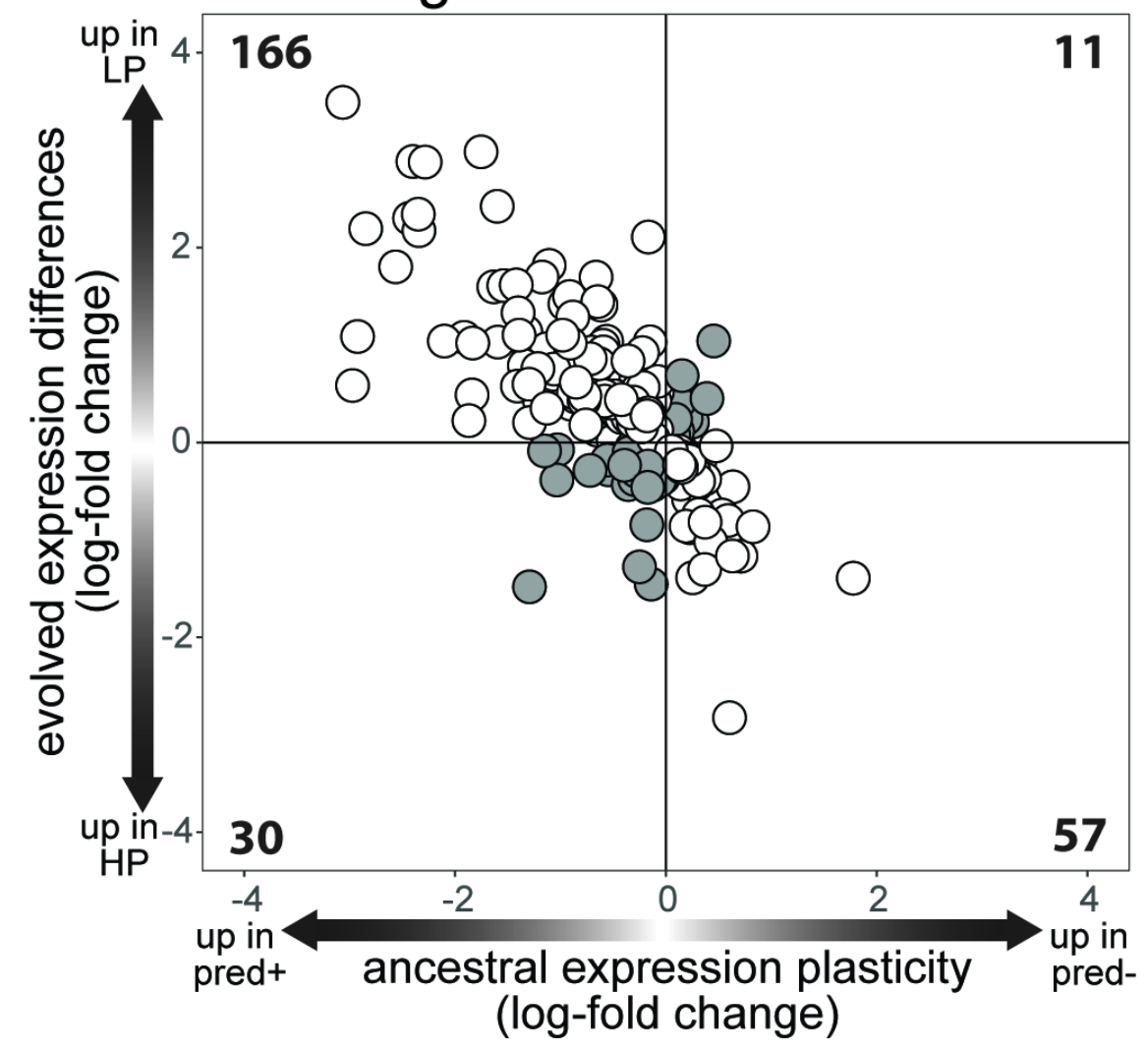

QUARE drainage

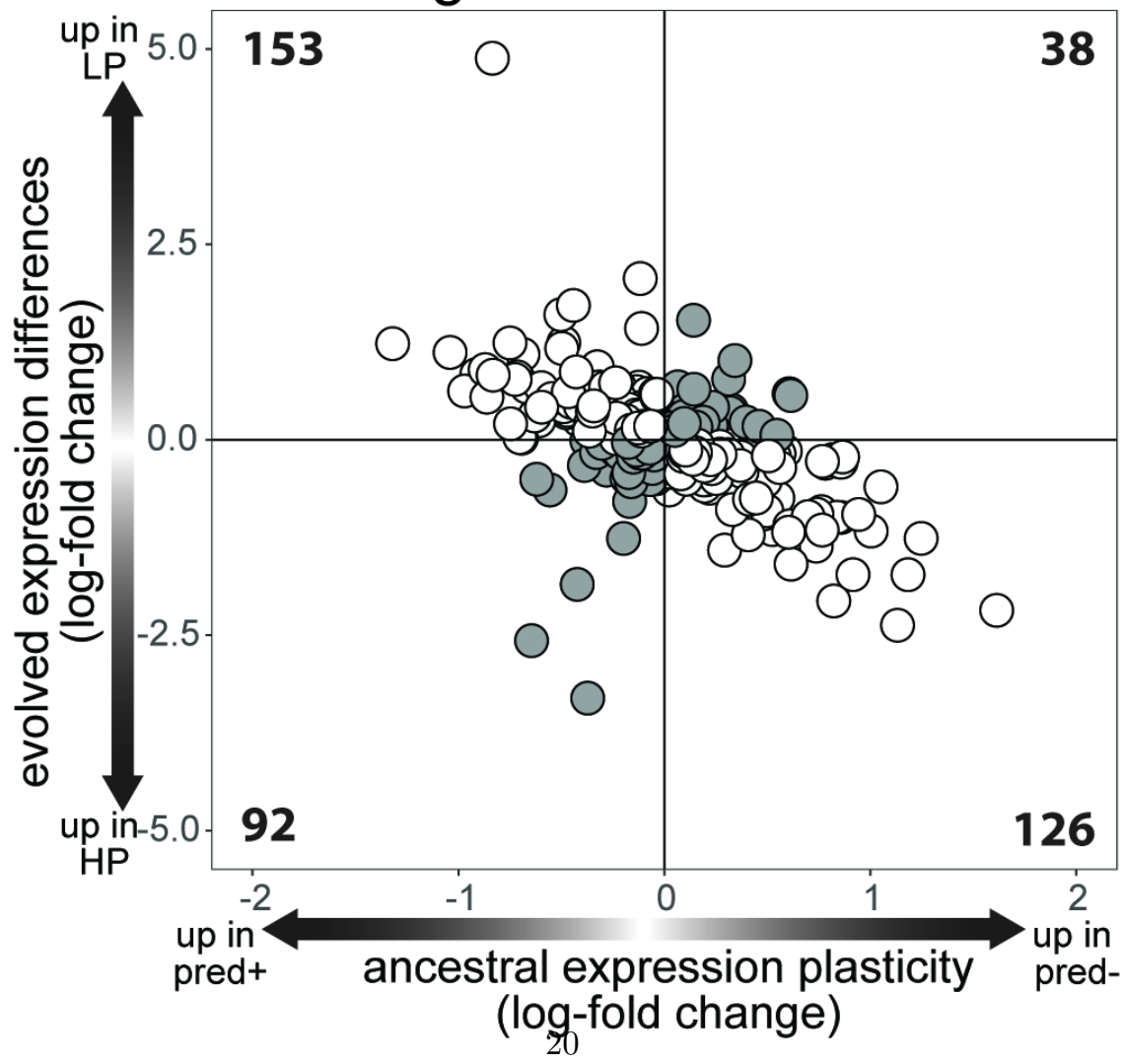


Figure 3. Relationship between ancestral plasticity and evolved expression divergence. Genes exhibiting significant ancestral expression plasticity (i.e. different between high-predation fish reared with and without predator cues) were more likely to also differ in expression between high- and low-predation populations reared without predator cue exposure in both the Aripo (top) and Quare (bottom) lineages. Each circle represents expression differences in a single transcript with statistically significant ancestral plasticity in the high-predation population as well as significant expression differences between high- and low-predation populations. Plastic expression changes were more likely to be in the opposite (white circles) than in the same (grey circles) direction as genetic expression divergence. The number of transcripts in each quadrant is indicated on the graphs. Note that axes differ between plots in order to best visualize variation within each dataset.

A
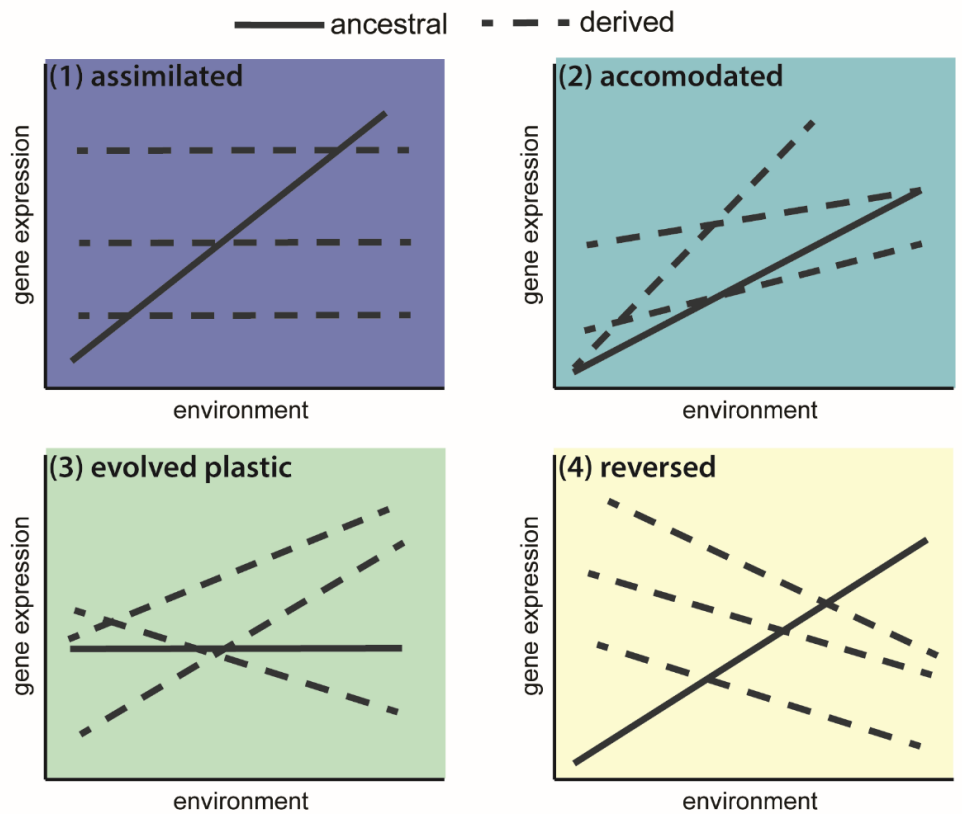

B ARIPO drainage

QUARE drainage
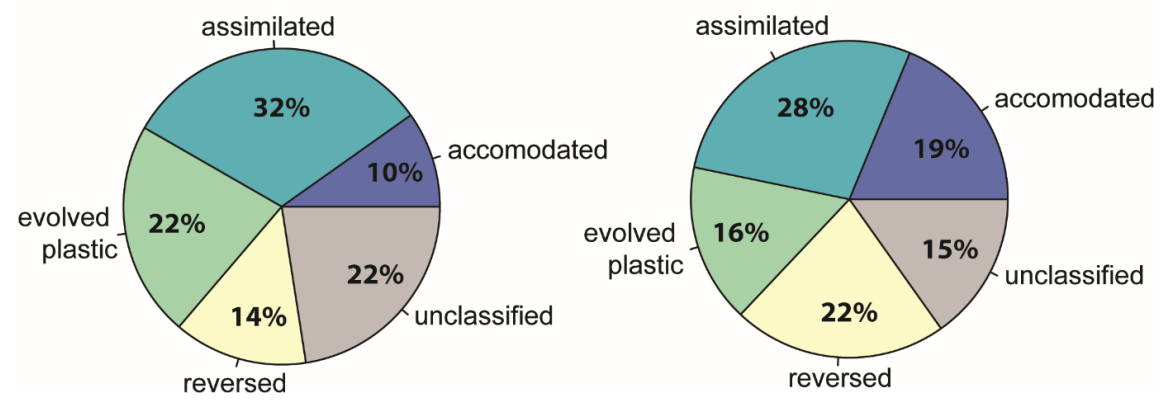

Figure 4. Evolution of transcript expression plasticity. (A) We grouped transcripts with significant interaction effects into one of four categories based on patterns of evolution in expression plasticity: (1) Assimilated: plasticity in the ancestral high-predation population but a loss of plasticity in the derived low-predation population; (2) Accommodated: a change in the degree, but no the direction, of plasticity in the derived as compared to the ancestral population; (3) Evolved plastic: no plasticity in the ancestral high-predation population but an emergence of plasticity in the derived low-predation population; (4) Reversed: opposing directions of plasticity in high- versus low-predation populations. We categorized remaining transcripts that had a significant main interaction effect, but no significantpost hoc rearing differences as 
unclassified. Adapted from Renn \& Schumer (2013). (B) All categories were represented in both Aripo and Quare datasets. 
POPULATION expression divergence

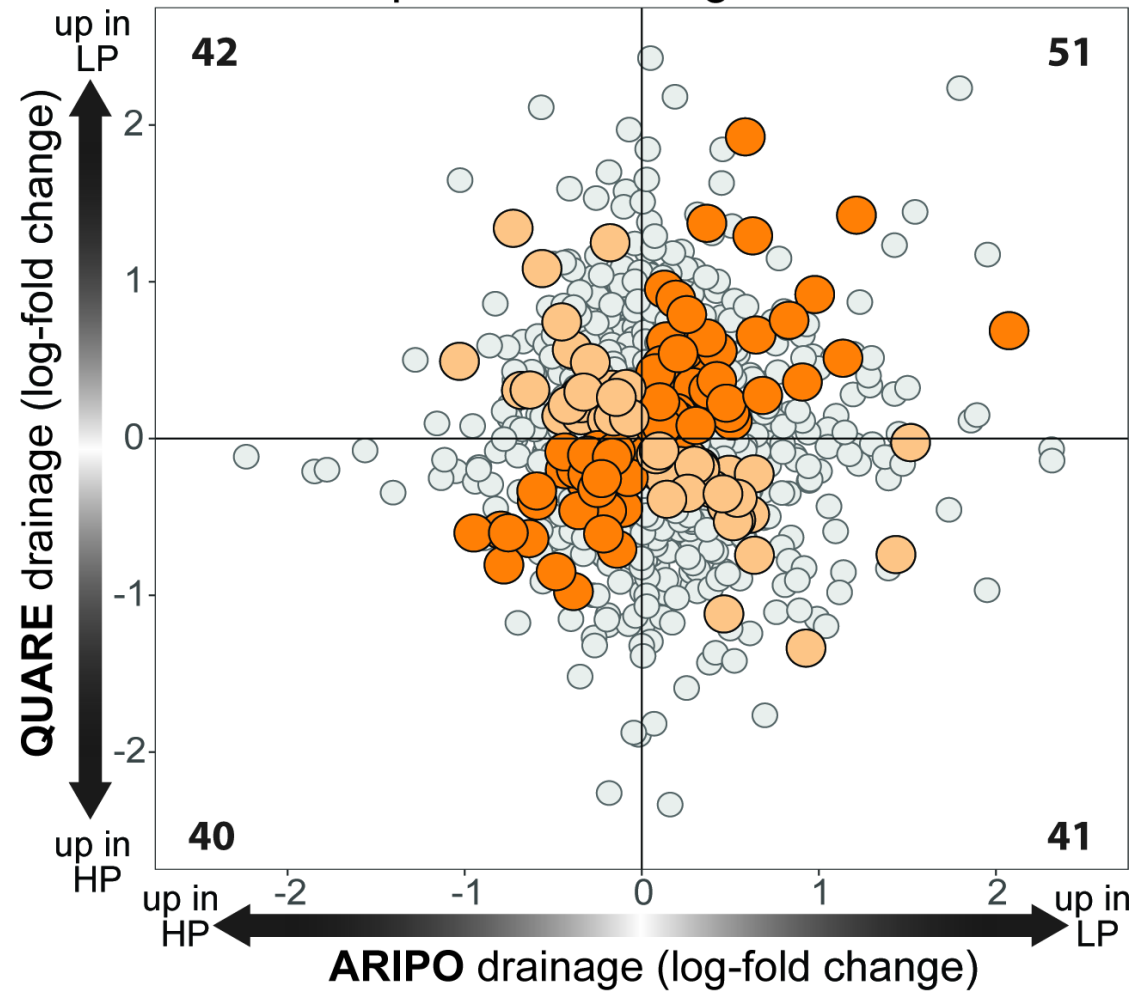

REARING expression differences

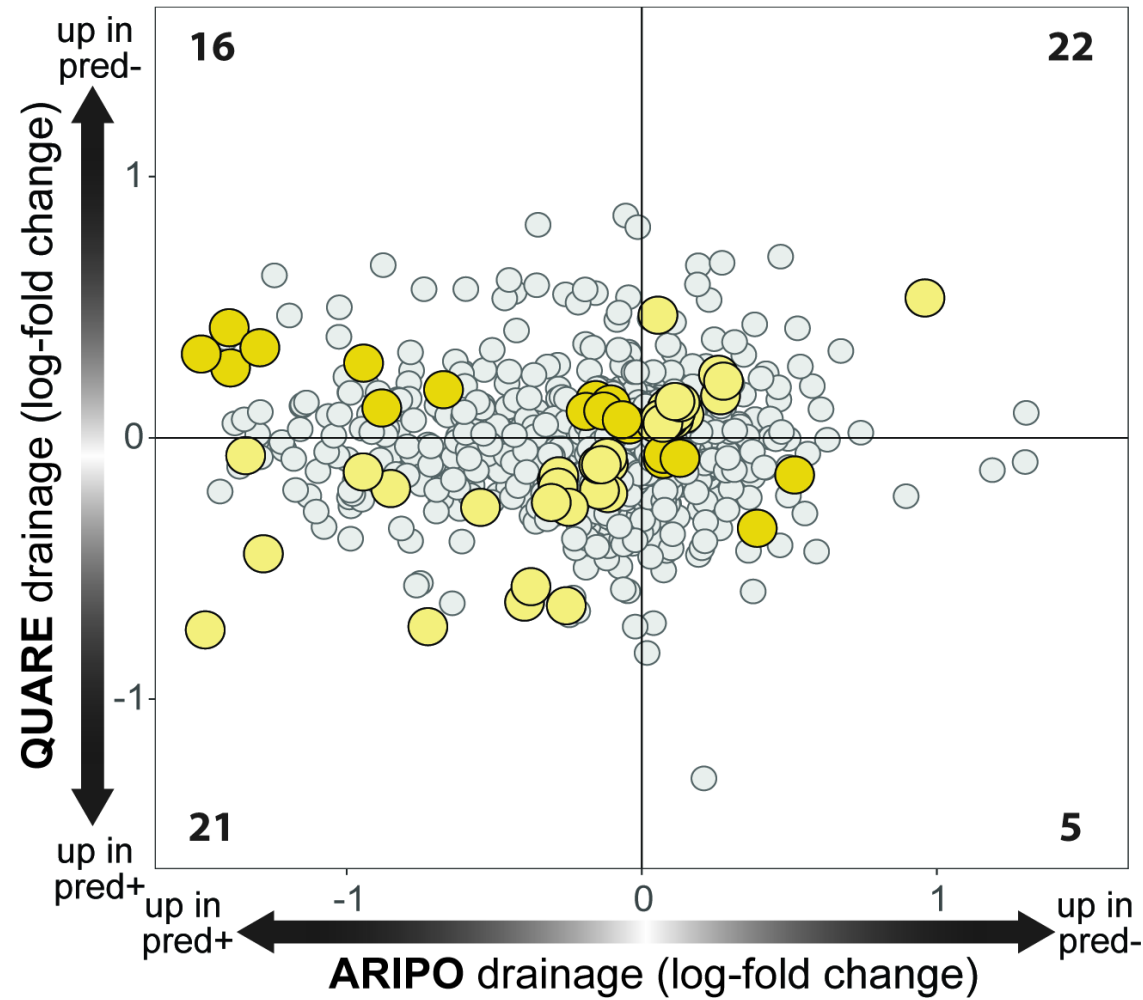


Figure 5. Overlap in population and rearing expression differences across evolutionary lineages. Of the transcripts differentially expressed based on population of origin (top), 124 were overlapping between drainages. Of these, 66 were concordantly differentially expressed (i.e. expression divergence in the same direction in both drainages; dark orange circles) and 58 were not (i.e. expression divergence in opposite directions between drainages; light orange circles), no different than expected by chance. Of the transcripts differentially expressed based on rearing environment (bottom), 35 were overlapping in the two drainages. Of these, 19 were concordantly differentially expressed (dark yellow circles) and six were not (light yellow circles), more non-concordance than expected by chance. The number of differentially expressed transcripts in each quadrant is indicated on the graphs and transcripts differentially expressed in one but not both drainages are shown in grey.

\section{Supplemental Materials}

Table S1. Transcripts filtered from the analysis following identification as retinal contaminants. Provided as Excel file.

Table S2 . Transcripts significantly differentially expressed between high- and low-predation populations in the Aripo drainage. Provided as Excel file.

Table S3 . Transcripts significantly differentially expressed between high- and low-predation populations in the Quare drainage. Provided as Excel file.

Table S4 . Results of GO enrichment analyses for population, rearing, and interaction effects in the Aripo drainage. Provided as Excel file.

Table S5 . Results of GO enrichment analyses for population, rearing, and interaction effects in the Quare drainage. Provided as Excel file.

Table S6 . Population DE transcripts overlapping between Aripo and Quare drainages. 185 transcripts were DE in both drainages, of these 89 had expression differences in the same direction in both datasets (concordant differential expression). Provided as Excel file.

Table S7 . Rearing DE transcripts overlapping between Aripo and Quare drainages. 16 transcripts were $\mathrm{DE}$ in both drainages, of these 7 had expression differences in the same direction in both drainages (i.e. concordant differential expression). Provided as Excel file.

Table S8 . Pst estimates for the Aripo and Quare drainages. 

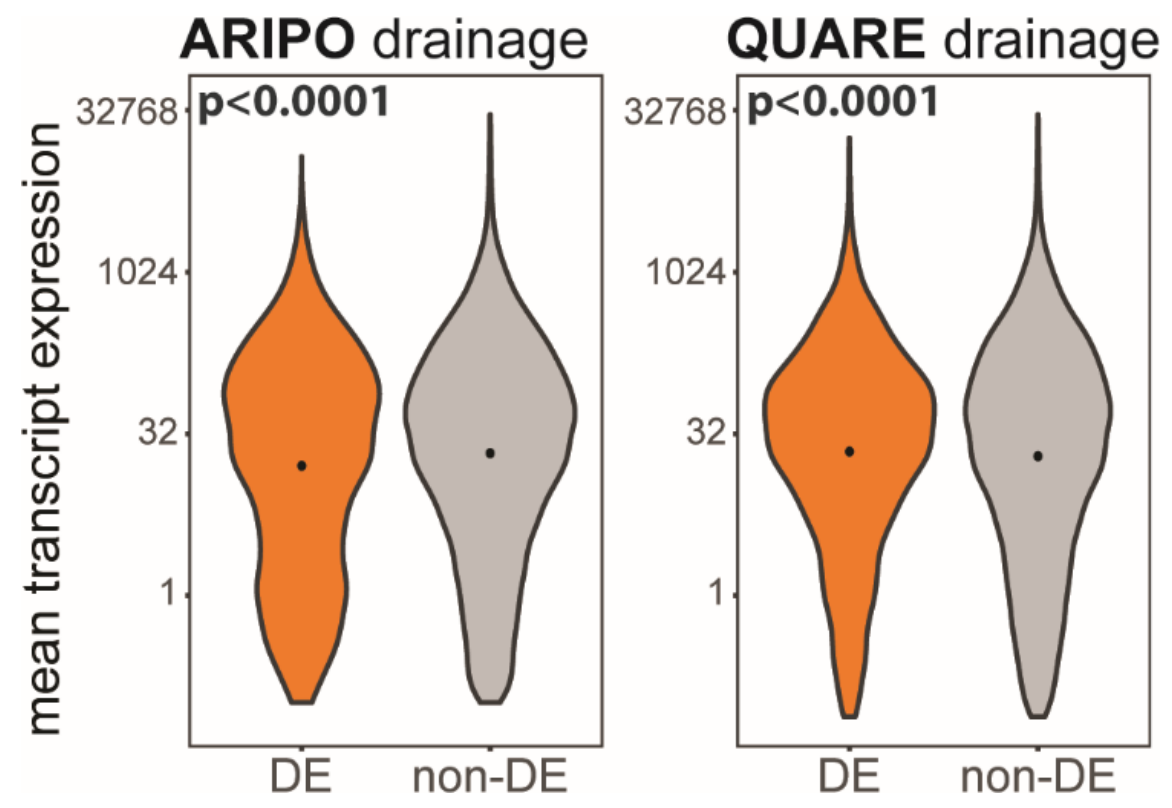

Figure S1. Differences in means but not variance of differentially expressed (DE) and non-differentially expressed (non-DE) transcripts. DE transcripts had significantly higher means in the Aripo drainage and lower means in the Quare drainage as compared to non-DE transcripts. Due to the large range of expression values, mean differences are plotted on a log scale (y-axis). 